\title{
Homeostatic Changes in GABA and Acetylcholine Muscarinic Receptors on GABAergic Neurons in the Mesencephalic Reticular Formation following Sleep Deprivation
}

\author{
${ }^{\circledR}$ Hanieh Toossi, ${ }^{\circledR}$ Esther del Cid-Pellitero, and ${ }^{\circledR}$ Barbara E. Jones
}

DOI:http://dx.doi.org/10.1523/ENEURO.0269-17.2017

Department of Neurology and Neurosurgery, Montreal Neurological Institute, McGill University, Montreal, Quebec H3A 2B4, Canada

\begin{abstract}
We have examined whether GABAergic neurons in the mesencephalic reticular formation (RFMes), which are believed to inhibit the neurons in the pons that generate paradoxical sleep (PS or REMS), are submitted to homeostatic regulation under conditions of sleep deprivation (SD) by enforced waking during the day in mice. Using immunofluorescence, we investigated first, by staining for c-Fos, whether GABAergic RFMes neurons are active during $S D$ and then, by staining for receptors, whether their activity is associated with homeostatic changes in $\mathrm{GABA}_{\mathrm{A}}$ or acetylcholine muscarinic type 2 (AChM2) receptors (Rs), which evoke inhibition. We found that a significantly greater proportion of the GABAergic neurons were positively stained for c-Fos after SD $(\sim 27 \%)$ as compared to sleep control (SC; $\sim 1 \%$ ) and sleep recovery (SR; $\sim 6 \%)$, suggesting that they were more active during waking with SD and less active or inactive during sleep with SC and SR. The density of GABA ${ }_{A} R s$ and AChM2Rs on the plasma membrane of the GABAergic neurons was significantly increased after SD and restored to control levels after SR. We conclude that the density of these receptors is increased on RFMes GABAergic neurons during presumed enhanced activity with $S D$ and is restored to control levels during presumed lesser or inactivity with $S R$. Such increases in $G_{A B A} R$ and $A C h M 2 R$ with sleep deficits would be associated with increased susceptibility of the wake-active GABAergic neurons to inhibition from GABAergic and cholinergic sleep-active neurons and to thus permitting the onset of sleep and PS with muscle atonia.
\end{abstract}

Key words: AChM2; GABAA; homeostasis; mice; waking

\section{Significance Statement}

Neuronal activity is regulated in a homeostatic manner such that prolonged activity results in increases in receptors to inhibitory neurotransmitters. Sleep-wake states are also regulated in a homeostatic manner likely through the regulation of sleep-wake regulatory neurons. Here, we established using c-Fos that GABAergic neurons in the mesencephalic reticular formation (RFMes), which are believed to inhibit pontine paradoxical sleep (PS or REMS) generating neurons, are active during enforced waking with sleep deprivation (SD). GABA $A_{A}$ and acetylcholine muscarinic type 2 receptors (AChM2Rs), which evoke inhibition, were increased on the membrane of the GABAergic neurons. The increase in these receptors would render the neurons more susceptible to inhibition by GABA and ACh and to thus permitting the onset of PS with muscle atonia.

Received August 1, 2017; accepted November 7, 2017; First published December 26, 2017.

The authors declare no competing financial interests.
Author contributions: H.T., E.d.C.-P., and B.E.J. designed research; E.d.C.-P. performed research; H.T. analyzed data; H.T. and B.E.J. wrote the paper. 


\section{Introduction}

Using transections and lesions, early studies established that the pontine tegmentum is critical for the generation of paradoxical sleep (PS or REMS) with muscle atonia (Jouvet, 1962). Using more localized lesions, subsequent studies indicated that the oral pontine $(\mathrm{PnO})$ reticular formation (RF) was most critical (Carli and Zanchetti, 1965) including a zone extending laterally and dorsally into the subcoeruleus (SubC) and sublaterodorsal tegmental (SubLDT) nuclei (Sakai, 1980; Hendricks et al., 1982; Friedman and Jones, 1984; Webster and Jones, 1988; Lu et al., 2006). Injections of the cholinergic agonist, carbachol, into this region elicited PS, presumably through excitation of PS generating neurons (George et al., 1964; Gnadt and Pegram, 1986; Baghdoyan et al., 1987; VanniMercier et al., 1989). Injections of glutamate agonists into the region even more potently elicited PS (Onoe and Sakai, 1995; Boissard et al., 2002). Moreover, neurons were identified there in immunohistochemical studies, which expressed c-Fos during PS rebound (Maloney et al., 1999, 2000) and in electrophysiological studies, which discharged during PS and are accordingly presumed to be PS-effector neurons (Sakai, 1980; Sakai and Koyama, 1996; Boucetta et al., 2014).

Multiple lines of evidence have indicated that PSeffector neurons in the pontine tegmentum are under inhibitory control through GABAergic input. Pharmacological studies showed that microinjection of bicuculline, a $\mathrm{GABA}_{A}$ receptor $\left(G A B A_{A} R\right)$ antagonist, into the $\mathrm{PnO}$ and adjacent areas of the pontine tegmentum elicited PS with muscle atonia (Xi et al., 1999; Boissard et al., 2002; Sanford et al., 2003). Early lesion studies had indicated that lesions of neurons within the mesencephalic tegmentum, particularly in the ventrolateral periaqueductal gray (VLPAG) and adjoining mesencephalic RF (RFMes; also called deep mesencephalic nucleus (DpMe; Paxinos and Franklin, 2001) produced marked hypersomnia with a prominent increase in PS (Petitjean et al., 1975; Lu et al., 2006). A PS increase was also evoked by microinjection of muscimol, a GABA $A$ agonist, and conversely, a PS decrease was evoked by microinjection of bicuculline, a $\mathrm{GABA}_{A} \mathrm{R}$ antagonist, into this region (Sastre et al., 1996), suggesting that GABAergic neurons located in the VLPAG and RFMes could potentially inhibit the PS-effector neurons in the pons to which they project (Sapin et al., 2009). The most effective site was claimed to be within the RFMes (or dorsocaudal central tegmental field in the cat) where direct excitation by glutamate agonists also pre-

This work was supported by the Canadian Institutes of Health Research Grant CIHR MOP-130502 (to B.E.J.).

Acknowledgements: We thank Lynda Mainville for her technical assistance and Anton Plaversuski for his contribution to the behavioral experiments.

Correspondence should be addressed to Barbara E. Jones, Montreal Neurological Institute, 3801 University Street, Montreal, Quebec H3A 2B4, Canada, E-mail: barbara.jones@mcgill.ca.

DOI:http://dx.doi.org/10.1523/ENEURO.0269-17.2017

Copyright (C) 2018 Toossi et al.

This is an open-access article distributed under the terms of the Creative Commons Attribution 4.0 International license, which permits unrestricted use, distribution and reproduction in any medium provided that the original work is properly attributed. vented PS and elicited waking (Crochet and Sakai, 1999). Indeed, GABAergic neurons are distributed through this field (Ford et al., 1995), and some expressed c-Fos with PS deprivation (Sapin et al., 2009). Thus, GABAergic neurons of the RFMes could possibly control the generation of PS by holding the PS-effector neurons in the pons under inhibition during waking. These neurons also appear to be controlled in turn by GABAergic inhibitory input through $\mathrm{GABA}_{A} \mathrm{Rs}$ (Sastre et al., 1996). They could also be inhibited by acetylcholine (ACh) through muscarinic type 2 (AChM2) receptors (AChM2Rs; Egan and North, 1986), which have been visualized on GABAergic RFMes neurons (Brischoux et al., 2008). Indeed, elicitation of PS by cholinergic agonists appears to be determined to an important degree by AChM2Rs (Velazquez-Moctezuma et al., 1989; Baghdoyan and Lydic, 1999), which could be associated with inhibition of wake-active GABAergic neurons. Thus it has been suggested that GABAergic neurons of the RFMes could be inhibited through $\mathrm{GABA}_{A} R$ and/or AChM2R by GABAergic and/or cholinergic PS-active neurons identified in the LDT, SubLDT, and SubC, to permit the occurrence of PS (Sastre et al., 1996; Lu et al., 2006; Brischoux et al., 2008; Boucetta et al., 2014).

Sleep is under homeostatic control, such that extended waking increases the propensity for sleep (Borbély et al., 1984). The activity of individual neurons is also homeostatically regulated (Turrigiano, 1999), whereby prolonged activity results in decreases in excitability and activity through increases in inhibitory and decreases in excitatory receptors (Turrigiano et al., 1998; Kilman et al., 2002; Marty et al., 2004). Previous in vitro work on cultured neurons has shown that increased activity of hippocampal neurons results in increases in the density of $\mathrm{GABA}_{A} \mathrm{Rs}$ on their plasma membrane (Marty et al., 2004). Similarly, in vivo studies of increased activity in hippocampal neurons induced by seizures showed increases in $\mathrm{GABA}_{\mathrm{A}} \mathrm{Rs}$ on the postsynaptic membrane associated with increases in IPSCs (Nusser et al., 1998). It would thus appear possible that presumed wake-active GABAergic RFMes neurons, which would normally control the occurrence of sleep and $\mathrm{PS}$, could undergo homeostatic regulation under conditions of prolonged activity, as would presumably occur in rodents with sleep deprivation (SD) during the day, when they normally sleep the majority of the time.

In this study, we aimed to determine in mice using immunohistochemistry first, by staining for c-Fos, whether RFMes GABAergic neurons were active following SD and second by staining for receptors, whether their prolonged activity was associated with changes in $\mathrm{GABA}_{\mathrm{A}} \mathrm{R}$ and/or $\mathrm{AChM} 2 \mathrm{Rs}$ in a manner indicative of homeostatic changes.

\section{Materials and Methods}

All procedures conformed to the guidelines of the $\mathrm{Ca}$ nadian council on animal care and were approved by the McGill University Animal Care Committee.

\section{SD and recovery experimental procedures}

A total number of 12 adult male mice (C57BL/6, 20-25 g) were received from the supplier (Charles River) and housed individually under a 12/12 h light/dark cycle (lights 
on from 7 A.M. to 7 P.M.) at $22^{\circ} \mathrm{C}$ ambient temperature and with unlimited access to food and water at all times. As described in detail in another manuscript by the authors (Del Cid-Pellitero et al., 2017), animals were maintained in their home cages for the duration of the experiment and recorded by video and telemetric EEG using HomeCageScan software (HomeCageScan 3.0; Clever Systems). For telemetric recording of the EEG, two electrodes were placed symmetrically over parietal cortex along with two for reference over cerebellum and were connected by wires to a transmitter (F20-EET; Data Sciences International, DSI) implanted subcutaneously along the flank. Following surgery, the mice were allowed one week to recover.

The three experimental groups were composed of: (1) sleep control (SC) mice allowed to sleep undisturbed for 2 $\mathrm{h}$ from $\sim 2$ to $\sim 4$ P.M. ( zeitgeber time, ZT 7-9; $n=3$ ), (2) sleep deprived mice (SD) maintained awake for $2 \mathrm{~h}(n=3)$ or $4 \mathrm{~h}(n=3)$ from $\sim 12$ to $\sim 4$ P.M. ( Z ZT 5-9), and (3) sleep recovery $(\mathrm{SR})$ mice allowed to sleep for $2 \mathrm{~h}$ from $\sim 2$ to $\sim 4$ P.M. ( $\sim$ ZT 7-9) after being maintained awake for 4 $\mathrm{h}$ before euthanasia $(n=3)$. The mice were maintained awake by gentle stimulation with a soft paintbrush of the whiskers each time the mouse appeared to be preparing to sleep. Mice were immediately anaesthetized after the experimental period at $\sim 4$ P.M. ( Z ZT 9) with sodium pentobarbital (Euthanyl, $100 \mathrm{mg} / \mathrm{kg}$; Bimeda-MTC) and perfused transcardially with $30 \mathrm{ml}$ of cold saline followed by $200 \mathrm{ml}$ of $3 \%$ paraformaldehyde solution. Brains were removed, postfixed in $3 \%$ paraformaldehyde for $1 \mathrm{~h}$ at $4^{\circ} \mathrm{C}$, then placed in $30 \%$ sucrose solution at $4^{\circ} \mathrm{C}$ for $2 \mathrm{~d}$, frozen to $-50^{\circ} \mathrm{C}$, and stored at $-80^{\circ} \mathrm{C}$.

Sleep and waking were scored by behavior and EEG using HomeCageScan software.

\section{Immunohistochemistry}

Coronal sections were cut through the brainstem on a freezing microtome at $20-\mu \mathrm{m}$ thickness and collected in five adjacent series, such that sections were separated by $100-\mu \mathrm{m}$ intervals in each series. Free floating sections were rinsed in $0.1 \mathrm{M}$ Trizma saline buffer $(\mathrm{pH} 7.4)$, then incubated in $6 \%$ normal donkey serum buffer for $30 \mathrm{~min}$ and subsequently incubated overnight at room temperature in a buffer containing $1 \%$ normal donkey serum with combinations of two primary antibodies: mouse anti-GAD67 (1:250, Millipore, catalog \#MAB5406, RRID: AB_2278725) or rabbit anti-GABA (1:2000, Sigma-Aldrich, catalog \#A2052, RRID: AB_477652) with rabbit anti-c-Fos (1:10,000, Oncogene, Millipore catalog \#PC38, RRID: AB_2106755), mouse anti-GABA ${ }_{A} R$ 32-3-chain [clone BD17, 1:100, Millipore (Millipore Bioscience Research Reagents), catalog \#MAB 341, RRID: AB_2109419], or rabbit anti-AChM2R (1:600, Sigma, catalog \#M9558, RRID: AB_260727). Subsequently, sections were incubated at room temperature for $2 \mathrm{~h}$ in appropriate combinations of cyanine-conjugated (Cy3 or Cy5) secondary antibodies from donkey (Jackson ImmunoResearch): Cy5-conjugated anti-mouse (1:800, catalog \#715-175-150, RRID: AB_2340819) or Cy5-conjugated anti-rabbit (1:800, catalog \#711-175-152, RRID: AB_2340607) with Cy3-conjugated anti-rabbit (1:1000, catalog \#711-165-152, RRID: AB_2307443) or Cy3- conjugated anti-mouse (1:1000, catalog \#715-165-150, RRID: AB_2340813). The $\mathrm{GABA}_{A} \mathrm{R} \quad \beta 2$-3-chain and $A C h M 2 R$ antibodies were produced and characterized years ago and have since been in use over many years (Levey et al., 1991; Fritschy and Mohler, 1995; Wan et al., 1997; Nusser et al., 1998). GABA and GAD67 antibodies used in this study were proven specific and have been used in previous immunohistochemical studies to identify the GABAergic neurons (Gonchar and Burkhalter, 1997; Brischoux et al., 2008). Sections were subsequently stained with green fluorescent Nissl stain (FNS; 1:2000, $\mathrm{N}-21480$, Invitrogen) for 20 min. Finally, sections were rinsed, mounted and coverslipped with glycerol (Fisher).

\section{Immunohistochemical image analysis}

Stained sections were viewed using a Leica DMLB microscope equipped with $\mathrm{x} / \mathrm{y} / \mathrm{z}$ motorized stage, a digital camera (Orca-R ${ }^{2}$, C10600-10B, Hamamatsu photonics K.K.) and fluorescence filters for excitation and emission of Cy2, Cy3, and Cy5 dyes. Images were acquired and analyzed using Stereolnvestigator software (MicroBrightField, MBF) and the Optical Fractionator Probe that permit unbiased, systematic random sampling of a region of interest for cell number estimation or measurement of specific parameters, including luminance. Given the application of systematic random sampling for the examination and marking of cells at high magnification and the subsequent measurement of fluorescence intensity of the receptor staining in the marked receptor-positive cells employed here, double blind procedures were not applied in this process. In each series, three sections (at 100- $\mu \mathrm{m}$ intervals) were taken through the RFMes. In each section, a contour was traced under a $5 \times$ objective around the RFMes (also called DpMe; Paxinos and Franklin, 2001; Fig. 1A). Multi-channel image stacks (with $0.5-\mu \mathrm{m}$ thickness for each optical section) were acquired under a $40 \times$ objective through the mounted histologic section of $\sim 15-\mu \mathrm{m}$ thickness. For the purpose of counting and further analysis, a grid size of $300 \times 300 \mu \mathrm{m}^{2}$ and a counting frame of $120 \times 120 \mu \mathrm{m}^{2}$ were used. Across the three sections, $\sim 21$ counting frames were acquired and analyzed per series. Within these images, all cells located below $1 \mu \mathrm{m}$ from the surface of the section were counted, thus through $14 \mu \mathrm{m}$ of the section. The average number of GAD-immunostained neurons counted across series on one side was $53.75 \pm 3.38$ (mean \pm SEM), and that of GABA-immunostained neurons was $115.91 \pm 8.09$, reflecting the different sensitivities of these antibodies or immunostaining for the synthetic enzyme versus the neurotransmitter for GABA. Counting was performed by moving through the $z$ plane to assess the labeling for the c-Fos in the nucleus and for $\mathrm{GABA}_{A} \mathrm{Rs}$ or AChM2Rs on the membrane of the GABAergic neurons within RFMes region. Estimated total numbers of double-labeled cells were computed for each series (c-Fos-GAD, GABA $A_{A}$ GABA, AChM2R-GAD) and expressed as $\%$ of estimated GABA + cell populations per series through the RFMes.

Luminance measurements were also performed in 10 cells per animal for $\mathrm{GABA}_{A} \mathrm{Rs}$ and $\mathrm{AChM} 2 \mathrm{Rs}$ on the images, which were acquired under the same gain and 
A RFMes

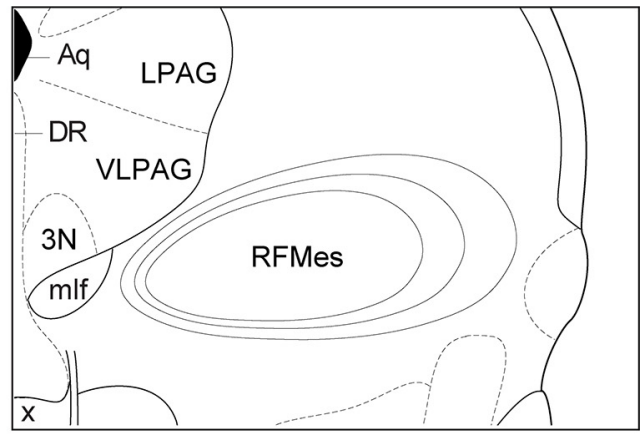

B GAD+o-c-Fos+/GAD+o

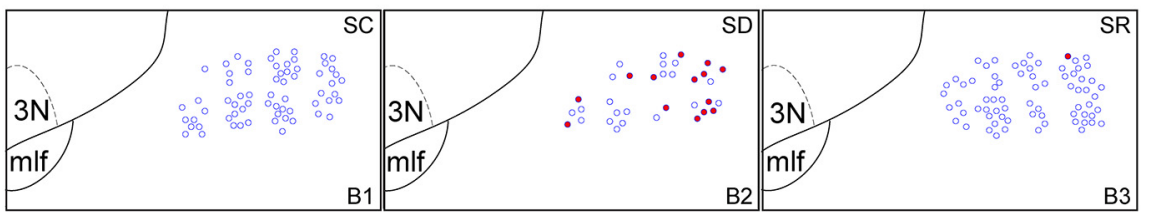

C. $G A B A+0-G A B A_{A} R+/ G A B A+0$

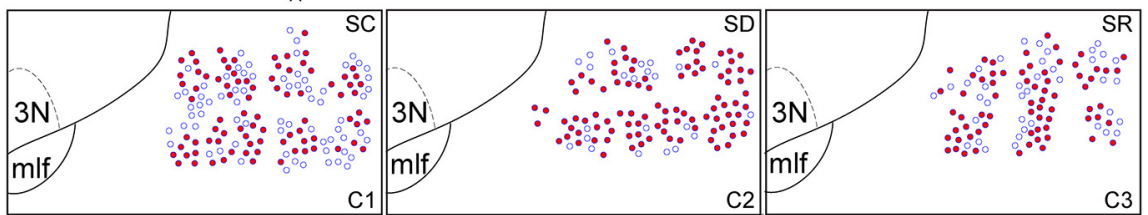

D. GAD+o-AChM2R+/GAD+o

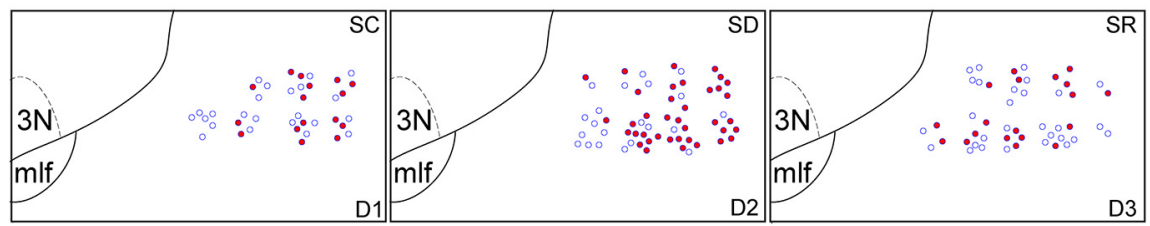

Figure 1. Atlas figure and distribution map of GABAergic neurons expressing c-Fos, GABAARs or AChM2Rs within the RFMes. $\boldsymbol{A}$, Atlas figure of the pontomesencephalic tegmentum showing the traced contours around the RFMes region in three adjacent sections separated by $100-\mu \mathrm{m}$ intervals, which were collapsed onto the middle section of this figure (bregma $-4.24 \mathrm{~mm}$; Paxinos and Franklin, 2001). B, Distribution map of the GAD+ (blue circle) and c-Fos +/GAD+ (red filled blue circle) neurons in an SC mouse brain (B1), an SD mouse brain (B2), and an SR mouse brain (B3). Note the greater number of c-Fos $+/ G A D+$ neurons in the SD mouse. $\boldsymbol{C}$, Distribution map of the GABA + (blue circle) and GABAAR +/GABA + (red filled blue circle) neurons in an SC mouse brain (C1), an SD mouse brain (C2), and an SR mouse brain (C3). Note the slightly higher number of GABAAR+/GABA+ neurons in the SD mouse. $\boldsymbol{D}$, Distribution map of the GAD+ (blue circle) and AChM2R+/GAD+ (red filled blue circle) neurons in an SC mouse brain (D1), an SD mouse brain (D2), and an SR mouse brain (D3). Note the greater number of AChM2R+/GAD+ neurons in the SD mouse. Scale bar: $1 \mathrm{~mm}$. 3N, oculomotor nucleus; Aq, aqueduct (Sylvius); DR. dorsal raphe nucleus; mlf, medial longitudinal fasciculus; $x$, decussation of the superior cerebellar peduncle.

exposure using Stereolnvestigator (above). They were acquired with the eight-bit setting of the digital camera, which thus provides converted gray scale images of the fluorescence with arbitrary units of 0-256 for luminance measures. Image acquisition was made as rapidly as possible for each cell to avoid bleaching of the fluorescence. As previously described in another article by the authors, a rectangular box sized at $1.5 \times 0.3 \mu \mathrm{m}^{2}$ was placed over the plasma membrane and another box over the nucleus for measurement of background staining in each cell. Subsequently, the luminance intensity of the nucleus was subtracted from that of the membrane in each cell.

Cell counts and luminance measurements were analyzed between experimental groups for GABAergic neurons and c-Fos or different type of receptors $\left(\mathrm{GABA}_{A}\right.$ or
AChM2) using one-way ANOVA followed by post hoc paired comparisons with Tukey's HSD correction for difference between groups (SYSTAT Software Inc., version 13). In an initial analysis, the proportion of c-Fos +/GAD+ neurons was found to differ significantly across the original four groups $\left(F_{(3,8)}=9.24, p=0.006\right)$; however, due to lack of significant differences between the SD2 and SD4 groups (post hoc paired comparisons, $p=$ $0.702)$, they were combined into one SD group in the current report.

Sections were also viewed by LSM 710 confocal laser scanning microscopy equipped with $\mathrm{Ar}$ 488-nm, He-Ne 543-nm, and He-Ne 633-nm lasers for excitation and emission of Cy2, Cy3, and Cy5 dyes. Images were acquired under a $63 \times$ oil objective with a 1.0 airy unit pinhole size for each channel. All figures were prepared 

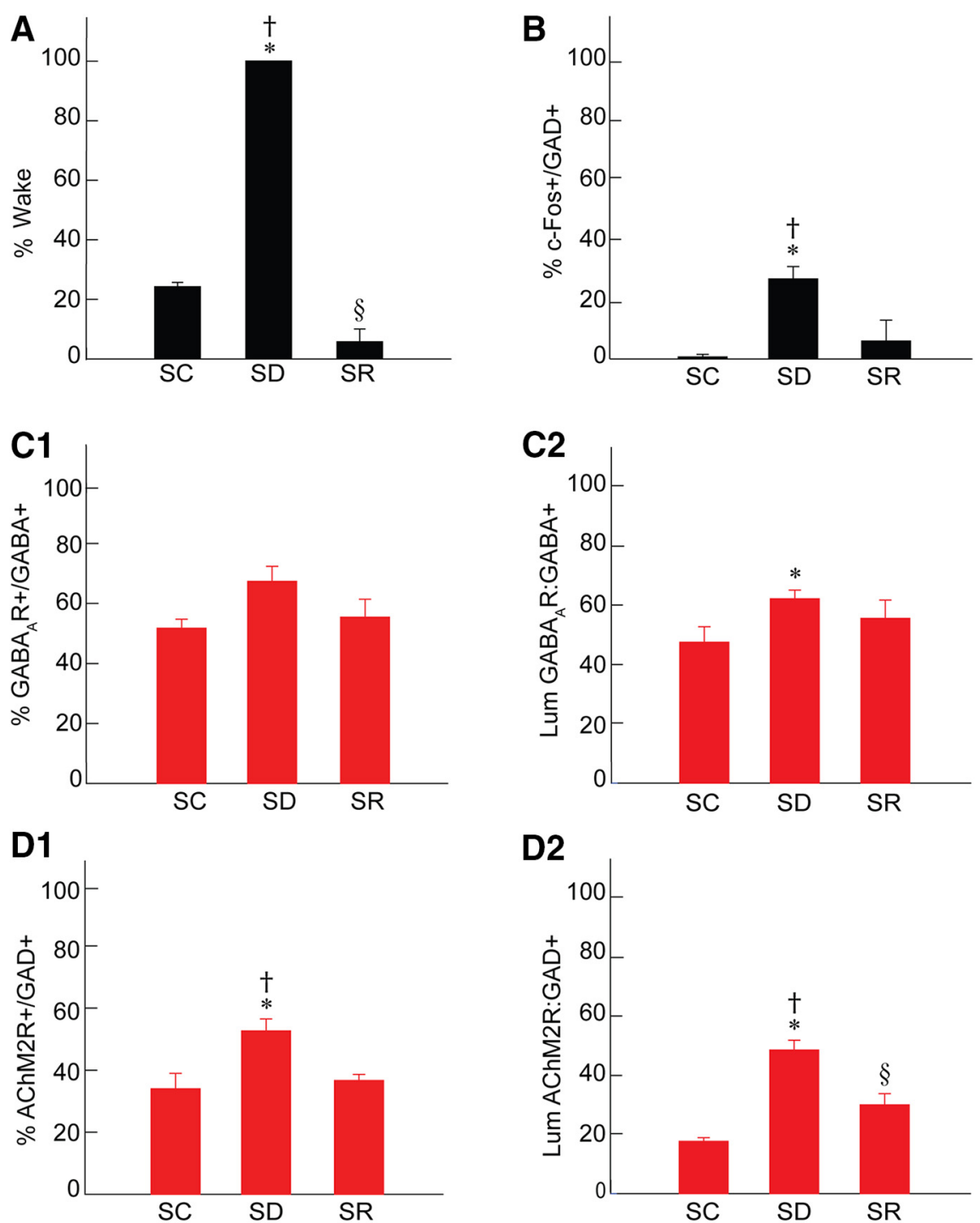

Figure 2. Sleep-wake states, c-Fos, GABA , and AChM2 receptors in RFMes GABAergic neurons across groups. $\boldsymbol{A}$, The percentage of time spent in wake during the $2 \mathrm{~h}$ preceding termination differed significantly across groups, being higher in SD as compared to SC and SR and lower in SR as compared to SC. B. The \% of GAD+ neurons that were positively immunostained for c-Fos (+) differed significantly between groups, being greater in SD as compared to SC and SR. $\boldsymbol{C}$, The \% of GABA+ neurons which were positively immunostained for the $\mathrm{GABA}_{A} R(+)$ increased insignificantly following $S D$ as compared to SC and SR (C1). The luminance of the $\mathrm{GABA}_{A} \mathrm{R}$ immunofluorescence on $\mathrm{GABA}_{A} \mathrm{R}+/ \mathrm{GABA}+$ neurons differed significantly, being higher in $\mathrm{SD}$ as compared to $\mathrm{SC}(\boldsymbol{C} 2)$. $\boldsymbol{D}$, The $\%$ of GAD+ neurons which were positively immunostained for AChM2R $(+)$ differed significantly between groups, being higher in SD as compared to SC and SR (D1). The luminance of the AChM2R immunofluorescence on AChM2R+/GAD+ neurons differed significantly, being higher in SD as compared to SC and SR (D2). Note that the changes in GABARs and AChM2Rs on RFMes GABAergic neurons parallel the changes in \% Wake and \% c-Fos +/GAD+ across groups; * indicates significant difference of SD relative to SC; † indicates significant difference of SD relative to SR; $\S$ indicates significant difference of SR relative to SC $(p<0.05)$, according to post hoc paired comparisons following one-way ANOVA (Table 1).

and composed using Adobe Creative Suite (CS4, Adobe System).

\section{Results}

\section{Sleep-wake states across groups}

Mice were prevented from falling asleep in the SD group $(n=6)$ and thereby maintained awake $\sim 100 \%$ of the time, whereas those in the SC group were awake $\sim 24 \%$ of the time $(23.79 \pm 1.02 \%$, mean \pm SEM, $n=3)$ and mice in the SR group $\sim 6 \%$ of the time $(5.67 \pm 2.63 \%$, $n=3$ ) during the $2 \mathrm{~h}$ before termination at $\sim 4$ P.M. (Fig. $2 A$; Table 1). Mice in the SR group were awake significantly less than those in the SC group. Being undisturbed, the mice in the SC group thus slept $\sim 76 \%$ of the time and mice in the SR group, which were allowed 2-h recovery after $4-h$ SD, slept $\sim 94 \%$ of the time, indicating a homeostatic response to SD. The major proportion of time for the SC and SR groups was spent in slow wave sleep (SWS; $66.93 \pm 1.71$ and $82.29 \pm 4.07 \%$, respectively), and a minor proportion in PS $(9.28 \pm 0.89$ and $12.03 \pm 0.87 \%$, 
Table 1. Summary of statistics

\begin{tabular}{|c|c|c|c|c|c|c|c|}
\hline \multirow[b]{2}{*}{ Dataset } & \multirow[b]{2}{*}{ Figure } & \multirow{2}{*}{$\begin{array}{l}\text { One-way ANOVA (group = three levels) } \\
\qquad F \text { value }\end{array}$} & \multirow{2}{*}{$\begin{array}{c}\mathrm{df} \\
\text { (group, error) }\end{array}$} & \multirow[b]{2}{*}{$p$ value } & \multicolumn{3}{|c|}{$\begin{array}{l}\text { Tukey's HSD paired } \\
\text { comparisons } p \text { value }\end{array}$} \\
\hline & & & & & SC-SD & SC-SR & SD-SR \\
\hline \% Wake & $1 \mathrm{~A}$ & 1381.12 & 2,9 & $<0.001$ & $<0.001 *$ & $<0.001 \S$ & $<0.001 \dagger$ \\
\hline$\%$ c-Fos/GAD+ & 1B & 12.97 & 2,9 & 0.002 & $0.003 *$ & 0.708 & $0.013 \dagger$ \\
\hline$\% \mathrm{GABA}_{\mathrm{A}} \mathrm{R}+/ \mathrm{GABA}+$ & $1 \mathrm{C} 1$ & 3.42 & 2,9 & 0.079 & 0.095 & 0.880 & 0.218 \\
\hline Lum GABA ${ }_{A} R: G A B A+$ & $1 \mathrm{C} 2$ & 3.04 & 2,117 & 0.051 & $0.042 *$ & 0.475 & 0.515 \\
\hline$\%$ AChM2R +/GAD + & 1D1 & 8.89 & 2,9 & 0.007 & $0.013 *$ & 0.893 & $0.028 \dagger$ \\
\hline
\end{tabular}

respectively). Both SWS and PS were significantly increased during SR relative to SC. With regard to behavior, increases in all waking behaviors occurred during SD, but most particularly still behavior, which reflected a quiet waking state (Del Cid-Pellitero et al., 2017).

\section{c-Fos expression in RFMes GABAergic neurons after SD}

Sections through the RFMes (Fig. 1A) were triplestained for Nissl (FNS), GAD, and c-Fos protein to assess changes in the activity of GABAergic neurons as a func-

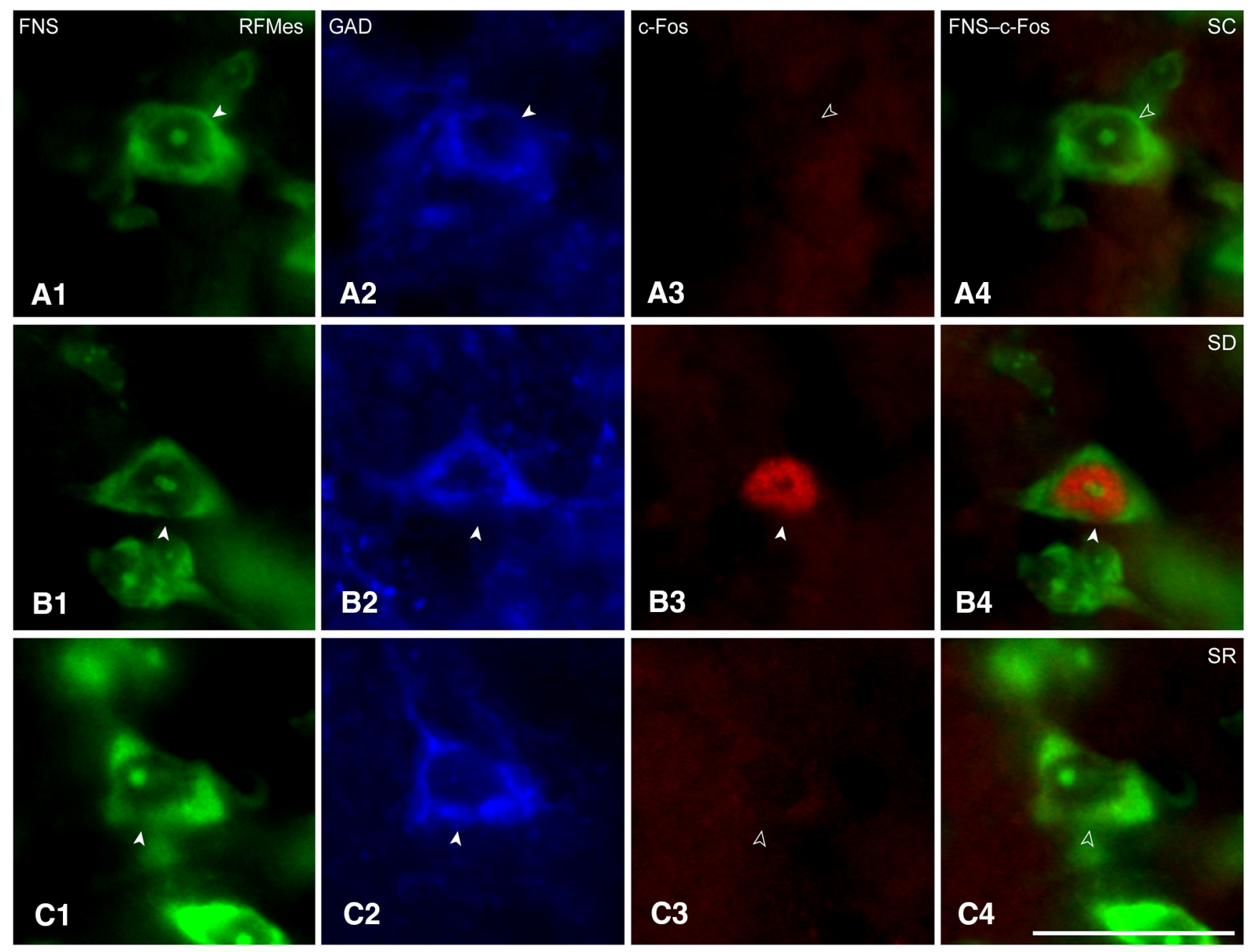

Figure 3. c-Fos in RFMes GABAergic neurons across groups. Fluorescent microscopic images show staining for Nissl with FNS (green; A1, B1, C1), immunostaining for GAD (blue; A2, B2, C2, with positive staining indicated by filled arrowheads), and immunostaining for c-Fos (red; A3, B3, C3, with positive staining indicated by filled arrowhead) along with dual staining for Nissl and c-Fos in merged images (green and red; $\boldsymbol{A 4}, \mathbf{B} 4, \mathbf{C} 4$, with positive c-Fos staining indicated by filled arrowhead). Note that c-Fos immunostaining is prominent in the nucleus of a GABAergic neuron from an SD mouse $(\mathbf{B 3}, \mathbf{B} 4)$, whereas it is not apparent in images from SC or SR mice $(\mathbf{A 3}, \boldsymbol{A 4}$ or $\mathbf{C 3}, \mathbf{C 4}$, indicated by open arrowheads). Scale bars: $20 \mu \mathrm{m}$. Image thickness: $500 \mathrm{~nm}$ in all panels. 


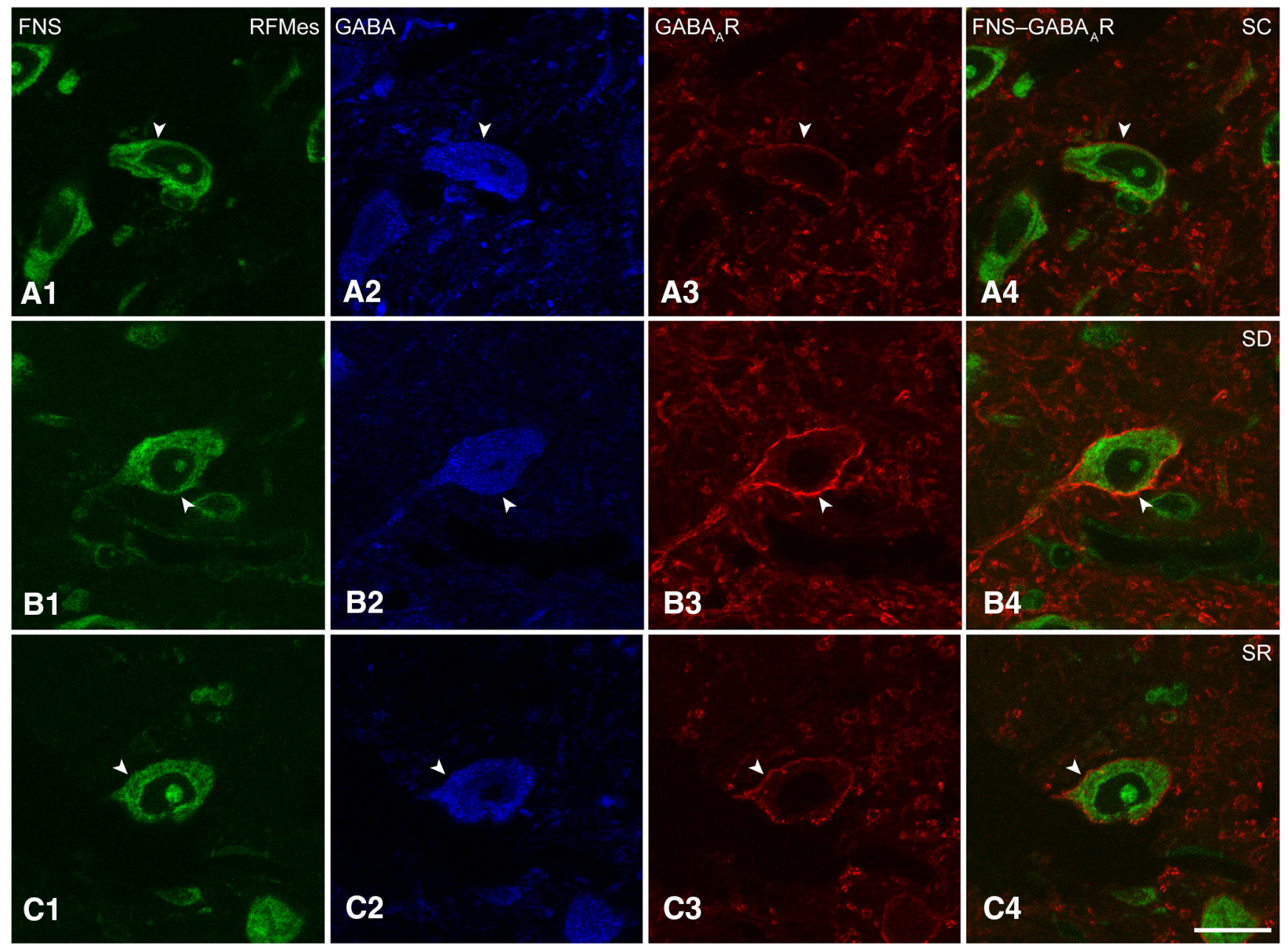

Figure 4. $G A B A_{A}$ Rs in RFMes GABAergic neurons across groups. Confocal microscopic images show all neurons stained for Nissl with FNS (green; $\boldsymbol{A 1}, \boldsymbol{B 1}, \boldsymbol{C 1}$ ), the GABAergic neurons immunostained for GABA (blue; $\boldsymbol{A 2}$, B2, C2, indicated by filled arrowhead), and for the $\mathrm{GABA}_{\mathrm{A}} \mathrm{Rs}$ in single (red; $\boldsymbol{A 3}, \boldsymbol{B} \mathbf{3}, \mathbf{C}$, indicated by filled arrowhead) and merged images $(\boldsymbol{A} 4, \mathbf{B} 4, \boldsymbol{C} 4$, indicated by filled arrowhead). Note that in an SC mouse, the $\mathrm{GABA}_{A} \mathrm{R}$ immunofluorescence is minimally visible, whereas in an $\mathrm{SD}$ mouse, it is prominent and bright. In an SR mouse, it appeared less bright than in the SD mouse. In all cases, the immunostaining is relatively continuous though with nonuniform intensity along the plasma membrane of the GABA+ neurons. Scale bars: $20 \mu \mathrm{m}$. Image thickness: $500 \mathrm{~nm}$ in all panels.

tion of enforced waking with SD as compared to SC and SR (Fig. $3 A-C$ ). c-Fos immunostaining was relatively rarely seen in the GAD+ neurons of the SC or SR mice, whereas it was prominent in the nucleus of multiple $\mathrm{GAD}+$ neurons of the SD mice (Figs. 1B1-B3, 3A-C). The average proportion of $\mathrm{C}-\mathrm{Fos}+/ \mathrm{GAD}+$ cells differed significantly between groups $(n=3$ or 6 mice per group; Fig. $2 B$; Table 1), being significantly greater in SD (26.93 \pm $3.41 \%)$ as compared to SC $(0.66 \pm 0.54 \%)$ and SR $(6.02$ $\pm 4.35 \%)$ groups. The \% c-Fos $+/ G A D+$ cells did not differ between SC and SR indicating that the proportion of c-Fos+/GAD+ neurons, returned to control or baseline levels following SR.

\section{GABA $_{A}$ Rs on RFMes GABAergic neurons after SD}

Triple-stained sections for FNS-GABA-GABA $A_{A} R$ were analyzed to assess the presence and intensity of $G A B A_{A} R s$ on RFMes GABAergic neurons in brains of mice from SC, SD, and $S R$ groups (Fig. $4 A-C$ ). GABA $A_{A} R$ immunostaining ap- peared to be primarily located on the plasma membrane of the GABA+ neurons. It varied in intensity among neurons and mice yet appeared to be consistently most intense in GABA+ neurons of the SD mice (Fig. $4 A-C$ ).

A major proportion of the GABA+ neurons in the RFMes appeared to be positively immunostained $(+)$ for the $\mathrm{GABA}_{A} R$ over the plasma membrane in all mice of all groups. However, the proportion of $\mathrm{GABA}_{A} \mathrm{R}+/ \mathrm{GABA}+$ neurons appeared to be somewhat greater in the $S D$ group as compared to SC and SR groups (Fig. 1C1-C3 ). The average proportion of $\mathrm{GABA}_{\mathrm{A}} \mathrm{R}+/ \mathrm{GABA}+$ neurons differed by a trend between groups ( $n=3$ or 6 mice per group; Fig. 2C1; Table 1) and was increased by a trend following SD $(67.33 \pm 4.14 \%)$ as compared to SC $(51.75$ $\pm 1.84 \%)$ but not as compared to SR $(55.42 \pm 3.69 \%)$. The $\% \mathrm{GABA}_{\mathrm{A}} \mathrm{R}+/ \mathrm{GABA}+$ neurons also did not differ between $S C$ and $S R$ indicating that the proportion of $\mathrm{GABA}_{\mathrm{A}} \mathrm{R}+/ \mathrm{GABA}+$ neurons returned toward control or baseline levels following SR. 


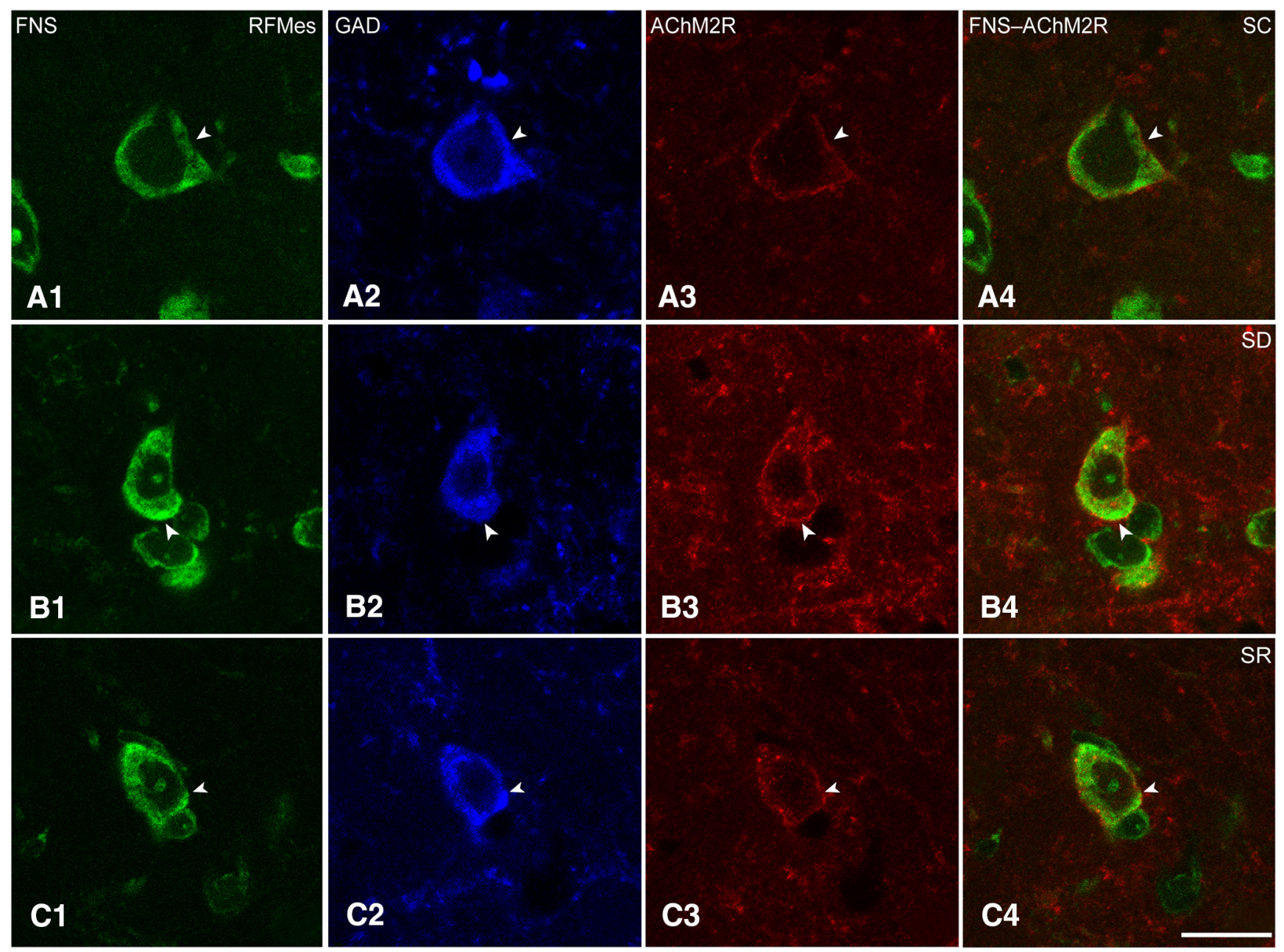

Figure 5. AChM2Rs in RFMes GABAergic neurons across groups. Confocal microscopic images show all neurons stained for Nissl with FNS (green; $\boldsymbol{A 1}, \boldsymbol{B 1}, \boldsymbol{C 1}$ ), the GABAergic neurons immunostained for GAD (blue; $\boldsymbol{A 2}, \mathbf{B 2}, \mathbf{C 2}$, indicated by filled arrowhead), and for the AChM2Rs in single (red; $\mathbf{A 3}, \mathbf{B 3}, \mathbf{C}$, indicated by filled arrowhead), and merged images (A4, B4, C4, indicated by filled arrowhead). Note that in an SC mouse, the AChM2R immunofluorescence is minimally visible along the plasma membrane, whereas in an SD mouse, the AChM2R staining is bright and clearly visible along the full membrane of the GAD+ neuron. In an SR mouse, the staining appeared less bright than in the SD mouse. Scale bars: $20 \mu \mathrm{m}$. Image thickness: $500 \mathrm{~nm}$ in all panels.

The luminance of the $G A B A_{A} R$ immunostaining on the plasma membrane of $\mathrm{GABA}_{A} \mathrm{R}+/ \mathrm{GABA}+$ cells was significantly different between groups $(n=30$ or 60 cells per group; Fig. 2C2; Table 1), being significantly higher in SD $(61.96 \pm 2.85)$ as compared to SC (47.35 \pm 5.01$)$ but not different as compared to SR (55.39 \pm 5.89$)$. The luminance did not differ between SC and SR indicating that the density of $\mathrm{GABA}_{\mathrm{A}} \mathrm{Rs}$ on the membrane of the GABA+ neurons returned to control or baseline levels following SR.

\section{AChM2Rs on RFMes GABAergic neurons after SD}

Sections triple-stained for FNS-GAD-AChM2R were analyzed to assess the presence and intensity of AChM2Rs on RFMes GABAergic neurons in brains of mice from SC, $\mathrm{SD}$ and SR groups (Fig. $5 A-C$ ). The AChM2R immunostaining was observed over the plasma membrane of the GAD+ neurons of all groups, although it appeared to be the most prevalent and dense over neurons of the SD group (Fig. 5A-C).
The proportion of $\mathrm{AChM} 2 \mathrm{R}+/ \mathrm{GAD}+$ neurons in the RFMes appeared to be somewhat higher in the SD group as compared to the SC and SR groups (Fig. 1D1-D3). The average proportion of the AChM2R+/GAD + neurons differed significantly between groups $(n=3$ or 6 mice per group; Fig. 2D1; Table 1), being significantly higher following SD $(52.63 \pm 3.17 \%)$ compared to SC $(34.00 \pm$ $3.05 \%)$ and SR $(36.66 \pm 1.21 \%)$. The \% AChM2R+/ GAD + did not differ between SC and SR indicating that proportion of AChM2R+/GAD+ neurons returned to control or baseline levels following SR.

The luminance of the AChM2R immunofluorescence over the membrane of the AChM2R+/GAD + cells was significantly different between groups ( $n=30$ or 60 cells per group; Fig. 2D2; Table 1), being significantly greater in the SD $(48.40 \pm 3.27)$ as compared to SC $(17.65 \pm 1.15)$ and SR groups $(29.91 \pm 2.84)$. The luminance measure was also significantly higher in SR as compared to SC indicating that the density of AChM2Rs on the membrane 
of the GAD+ neurons did not fully return to the baseline control level with SR.

\section{Discussion}

The present results show that RFMes GABAergic neurons express c-Fos with SD and are thus likely more active during enforced waking during the day, when rodents normally sleep the majority of the time. Immunohistochemical images also show increases in $G_{A B A} R$ and AChM2R on the RFMes GABAergic neurons with SD, suggesting that with increased activity during enforced waking, the neurons are regulated in a homeostatic manner by increases in receptors which elicit inhibition.

\section{c-Fos expression in RFMes GABAergic neurons following SD}

In triple-stained sections through the RFMes, c-Fos immunostaining was evident in the nucleus of multiple GAD + neurons in the SD group, whereas it was seen in few or no GAD+ neurons in the SC and SR groups. Somewhat different results were reported in rats following selective and prolonged (72 h) PS deprivation, which was associated with increased c-Fos in VLPAG/DpMes GAD+ neurons with both SD and SR as compared to SC (Sapin et al., 2009). Here using total and short (2-4 h) SD, the average proportion of c-Fos $+/ G A D+$ neurons significantly increased only following SD as compared to SC and returned to control levels with SR. Similar increases in c-Fos expression have been documented following SD as compared to SC and SR in orexin (Orx) neurons, which from electrophysiological recordings, are known to discharge during waking and to be silent during sleep (Lee et al., 2005; Modirrousta et al., 2005). In contrast, significantly less c-Fos expression was found following SD as compared to $\mathrm{SR}$ in melanin concentrating hormone $(\mathrm{MCH})$ neurons, which are known from recordings to be silent during waking and discharge during sleep (Modirrousta et al., 2005; Hassani et al., 2009). We thus interpret our results to suggest that RFMes GABAergic neurons are active during enforced waking with SD and possibly not active or less active during SWS and PS with SC and SR. However, such assumptions can only be proven by electrophysiological recordings of identified GABAergic neurons in the RFMes.

\section{Homeostatic regulation of RFMes GABAergic neurons through GABA ${ }_{A}$ ss}

In triple-stained sections, $\mathrm{GABA}_{\mathrm{A}}$ Rs were apparent over the plasma membrane in a relatively large proportion of RFMes GABA + neurons, which was the greatest following enforced waking with SD. The fluorescence intensity of the $G A B A_{A} R s$ was significantly higher on the membrane of the GABA+ neurons following enforced waking and presumed increased activity with $S D$ as compared to $\mathrm{SC}$ or SR. The increases in GABA ${ }_{A} R$ s on RFMes neurons are similar to those seen on Orx and motor 5 (Mo5) neurons following enforced waking with SD, when these neurons have also been shown to be active according to c-Fos expression and/or electrophysiological recordings (Toossi et al., 2016, 2017). They are also similar to increases in $\mathrm{GABA}_{A}$ Rs seen on cholinergic basal forebrain neurons and cortical excitatory neurons following enforced waking and cortical activation with SD (Modirrousta et al., 2007; Del Cid-Pellitero et al., 2017). Collectively, these studies suggest that many cell groups which are normally active during waking and in a relative resting state during sleep undergo homeostatic regulation when submitted to enforced waking during the period when rodents normally sleep the majority of the time. Homeostatic changes associated with sleep and waking would include changes in glutamate as well as GABARs, which have been documented in the cortex and for which hypotheses and conclusions concerning Hebbian synaptic plasticity versus homeostatic synaptic scaling have diverged among scientists, and results have differed depending on the specific experimental paradigms and techniques employed (Tononi and Cirelli, 2003; Vyazovskiy et al., 2008; Aton et al., 2009; Xie et al., 2016; Del Cid-Pellitero et al., 2017; Diering et al., 2017; Puentes-Mestril and Aton, 2017; Timofeev and Chauvette, 2017).

Changes in $\mathrm{GABA}_{\mathrm{A}}$ Rs seen here can be likened to those seen in in vitro and in vivo studies, in which increased activity induced pharmacologically or by seizures resulted in increased $G_{A B A} R s$ on the plasma membrane and correlated increases in IPSCs (Nusser et al., 1998; Marty et al., 2004). Such homeostatic changes in receptors have been shown to occur within short time periods (Yamanaka et al., 2000; Ibata et al., 2008; Xie et al., 2016), similar to the period of SD employed here. The GABA ${ }_{A}$ Rs on the membrane of the RFMes GABA+ neurons returned to control levels here during increased sleep with SR, when according to the lack of c-Fos expression, the neurons were likely less active or inactive. Similar decreases of $\mathrm{GABA}_{\mathrm{A}} \mathrm{Rs}$ on the membrane occurred with SR in Orx neurons, when these neurons are known to be silent (Lee et al., 2005), whereas increases of $\mathrm{GABA}_{\mathrm{A}} \mathrm{Rs}$ occurred with $\mathrm{SR}$ on $\mathrm{MCH}$ neurons, when these neurons are known to discharge (Hassani et al., 2009; Toossi et al., 2016). Such changes can be likened to decreases in $G_{A B A} R$ clusters on the membrane of cultured neurons after pharmacologically induced periods of inactivity, which are associated with decreases in IPSCs (Kilman et al., 2002; Marty et al., 2004). The current results thus suggest homeostatic regulation through the $\mathrm{GABA}_{A} \mathrm{Rs}$ that would be associated with decreases in excitability following prolonged activity with SD and restorative return to baseline excitability following inactivity with SR.

Pharmacological evidence suggests that GABAergic neurons within the VLPAG and adjacent region of RFMes (or DpMe), which project to the pontine tegmentum, could be responsible for holding PS-effector neurons under inhibition during waking (Sastre et al., 1996; Xi et al., 1999; Boissard et al., 2002; Sanford et al., 2003). This suggestion was recently confirmed in an optogenetic study showing that selective activation of GABAergic neurons within the VLPAG region suppressed the onset of PS or shortened the duration of PS episodes (Weber et al., 2015). Optogenetic evidence was also presented to indicate that GABAergic neurons in the ventral medulla discharge during PS and could inhibit the presumed wakeactive VLPAG GABAergic neurons to promote PS (Weber 
et al., 2015). GABAergic neurons have also been recorded in the LDT/SubLDT and adjoining medial pedunculopontine tegmental (PPT) nuclei, which discharge maximally during PS and could thus possibly participate in the inhibition of $\mathrm{GABA}_{\mathrm{A}} \mathrm{R}$-bearing RFMes GABAergic neurons during PS (Boucetta et al., 2014). In addition, there are PS-active GABAergic neurons in the hypothalamus which project into the VLPAG/RFMes region and appear to inhibit GABAergic wake-active neurons therein (Hassani et al., 2010; Clément et al., 2012). Some of these PSactive GABAergic neurons, which project to the VLPAG/ RFMes region, also contain and use $\mathrm{MCH}$ (Hassani et al., 2009; Hassani et al., 2010; Clément et al., 2012). MCH neurons have been shown in electrophysiological recordings to discharge during sleep and PS (Hassani et al., 2009) and in optogenetic studies to enhance sleep with PS when stimulated, in part through release of GABA (Jego et al., 2013; Konadhode et al., 2013; Tsunematsu et al., 2014).

Here, we present evidence for the first time that presumed wake-active GABAergic neurons in the RFMes could undergo homeostatic plasticity with decreased excitability following increased activity with SD through increases in $\mathrm{GABA}_{\mathrm{A}} \mathrm{Rs}$ over their plasma membranes. They would accordingly be more sensitive to inhibitory input from sleep-active GABAergic neurons of the pons, medulla or hypothalamus and thus susceptible to permitting the onset of sleep following SD or deficiency.

\section{Homeostatic regulation of RFMes GABAergic neurons through AChM2Rs}

Similar to the distribution of $\mathrm{GABA}_{\mathrm{A}} \mathrm{Rs}, \mathrm{AChM} 2 \mathrm{Rs}$ were apparent over the plasma membrane in a proportion of RFMes GAD+ neurons across groups. Following SD, the proportion and luminance of the AChM2Rs on the plasma membrane of the RFMes GAD+ neurons increased relative to SC, presumably due to prolonged activity of RFMes GABAergic neurons during enforced waking. The proportion and density of the AChM2Rs on GAD+ neurons returned to control level with $S R$, presumably due to less activity or inactivity of the GABAergic neurons following SR. Our results here are parallel to the increase in proportion and density of AChM2Rs seen on the membrane of the Mo5 neurons following SD with prolonged waking, when these neurons are also presumed to be active (Toossi et al., 2017).

It was shown in pharmacological studies that microinjection of carbachol into the pontomesencephalic tegmentum, though most effectively into the pontine tegmentum, elicits PS with muscle atonia (George et al., 1964; Gnadt and Pegram, 1986; Baghdoyan et al., 1987; Vanni-Mercier et al., 1989). The induction of PS by administration of cholinergic agonists delivered into the pontomesencephalic tegmentum is dependent on AChM2Rs (Velazquez-Moctezuma et al., 1989; Baghdoyan and Lydic, 1999), which mediate hyperpolarization and inhibition (Egan and North, 1986). It can thus be posited that neurons which normally inhibit PS likely express AChM2Rs on their membrane. Given the immunohistochemical evidence for the presence of AChM2Rs on the membrane of
RFMes GABAergic neurons (Brischoux et al., 2008), it is suggested that ACh could inhibit RFMes GABAergic neurons through their AChM2Rs. Juxtacellular recordings and labeling of cholinergic neurons within the LDT/SubLDT/ PPT show that they are maximally active during wake and PS (Boucetta et al., 2014). Thus, cholinergic neurons could exert an inhibitory influence on neurons through AChM2Rs during PS, but would also do so during waking. Yet some of these GABAergic RFMes neurons were found to also bear Orx2Rs (Brischoux et al., 2008), and selective lesions of the Orx2R-bearing GABAergic neurons in this region were found to increase PS (Kaur et al., 2009). The AChM2Rs on the RFMes GABAergic neurons could normally be in balance with Orx2Rs, such that the excitatory action of Orx on the GABAergic neurons would normally prevail during waking, when Orx neurons discharge (Lee et al., 2005), whereas the inhibitory action of ACh would prevail in the absence of Orx, as during sleep and PS in normal cases or during narcolepsy with cataplexy in abnormal cases (Nishino and Mignot, 1997, 2011). With an increase of AChM2Rs on the GABAergic neurons with SD shown here, the inhibitory action of ACh could possibly also prevail during waking. Inhibition of RFMes GABAergic neurons by ACh would remove the GABAergic inhibition imposed by these neurons on PS-effector neurons located within the pontine tegmentum and permit the occurrence of PS or possibly increase the probability of narcolepsy with cataplexy, as occurs in patients under conditions of sleep deficits (Nishino and Mignot, 1997, 2011). Here, we present evidence that RFMes GABAergic neurons could undergo homeostatic plasticity with decreases in excitability following increased activity with SD through increases in AChM2Rs and resulting increased susceptibility to permitting sleep and PS with muscle atonia.

We conclude that RFMes GABAergic neurons are homeostatically regulated across the sleep-waking cycle through $G_{A B A} R$ and $A C h M 2 R$. The density of these receptors increases on the membrane of RFMes GABAergic neurons due to increased activity during enforced waking with SD, which would result in increased responsiveness of the neurons to inhibition by GABA and ACh. Their density is restored to baseline levels with SR when the neurons are likely inactive, which would result in the return of excitability and activity of the RFMes GABAergic neurons to stable levels. The homeostatic regulation of these presumed wake-active, PS-gating RFMes GABAergic neurons would in turn be paralleled by the similar regulation of their afferent wake-active Orx neurons, which would normally excite them, and the inverse regulation of their afferent PS-active MCH/GABAergic neurons, which would normally inhibit them (Toossi et al., 2016). The homeostatic changes in the different sleepwake regulatory neurons and circuits (Jones, 2017) likely underlie the homeostatic regulation of sleep-wake states.

\section{References}

Aton SJ, Seibt J, Dumoulin M, Jha SK, Steinmetz N, Coleman T, Naidoo N, Frank MG (2009) Mechanisms of sleep-dependent consolidation of cortical plasticity. Neuron 61:454-466. CrossRef Medline 
Baghdoyan HA, Lydic R (1999) M2 muscarinic receptor subtype in the feline medial pontine reticular formation modulates the amount of rapid eye movement sleep. Sleep 22:835-847. Medline

Baghdoyan HA, Rodrigo-Angulo ML, McCarley RW, Hobson JA (1987) A neuroanatomical gradient in the pontine tegmentum for the cholinoceptive induction of desynchronized sleep signs. Brain Res 414:245-261. Medline

Boissard R, Gervasoni D, Schmidt MH, Barbagli B, Fort P, Luppi PH (2002) The rat ponto-medullary network responsible for paradoxical sleep onset and maintenance: a combined microinjection and functional neuroanatomical study. Eur J Neurosci 16:1959-1973. Medline

Borbély AA, Tobler I, Hanagasioglu M (1984) Effect of sleep deprivation on sleep and EEG power spectra in the rat. Behav Brain Res 14:171-182. Medline

Boucetta S, Cisse Y, Mainville L, Morales M, Jones BE (2014) Discharge profiles across the sleep-waking cycle of identified cholinergic, GABAergic, and glutamatergic neurons in the pontomesencephalic tegmentum of the rat. J Neurosci 34:4708-4727. CrossRef

Brischoux F, Mainville L, Jones BE (2008) Muscarinic-2 and orexin-2 receptors on GABAergic and other neurons in the rat mesopontine tegmentum and their potential role in sleep-wake state control. $J$ Comp Neur 510:607-630. CrossRef Medline

Carli G, Zanchetti A (1965) A study of pontine lesions suppressing deep sleep in the cat. Arch Ital Biol 103:751-788. Medline

Clément $\mathrm{O}$, Sapin E, Libourel PA, Arthaud S, Brischoux F, Fort P, Luppi PH (2012) The lateral hypothalamic area controls paradoxical (REM) sleep by means of descending projections to brainstem GABAergic neurons. J Neurosci 32:16763-16774. CrossRef Medline

Crochet S, Sakai K (1999) Effects of microdialysis application of monoamines on the EEG and behavioural states in the cat mesopontine tegmentum. Eur J Neurosci 11:3738-3752. Medline

Del Cid-Pellitero E, Plavski A, Mainville L, Jones BE (2017) Homeostatic changes in GABA and glutamate receptors on excitatory cortical neurons during sleep deprivation and recovery. Front Syst Neurosci 11:17. CrossRef Medline

Diering GH, Nirujogi RS, Roth RH, Worley PF, Pandey A, Huganir RL (2017) Homer1a drives homeostatic scaling-down of excitatory synapses during sleep. Science 355:511-515. CrossRef Medline

Egan TM, North RA (1986) Acetylcholine hyperpolarizes central neurones by acting on an $M_{2}$-muscarinic receptor. Nature 319:405407. CrossRef Medline

Ford B, Holmes C, Mainville L, Jones BE (1995) GABAergic neurons in the rat pontomesencephalic tegmentum: codistribution with cholinergic and other tegmental neurons projecting to the posterior lateral hypothalamus. J Comp Neur 363:177-196. CrossRef Medline

Friedman L, Jones BE (1984) Computer graphics analysis of sleepwakefulness state changes after pontine lesions. Brain Res Bull 13:53-68. Medline

Fritschy JM, Mohler H (1995) $\mathrm{GABA}_{\mathrm{A}}$-receptor heterogeneity in the adult rat brain: differential regional and cellular distribution of seven major subunits. J Comp Neur 359:154-194. CrossRef Medline

George R, Haslett W, Jenden D (1964) A cholinergic mechanism in the brainstem reticular formation: induction of paradoxical sleep. Int J Neuropharmacol 3:541-552. Medline

Gnadt JW, Pegram GV (1986) Cholinergic brainstem mechanisms of REM sleep in the rat. Brain Res 384:29-41. Medline

Gonchar Y, Burkhalter A (1997) Three distinct families of GABAergic neurons in rat visual cortex. Cereb Cortex 7:347-358. Medline

Hassani OK, Lee MG, Jones BE (2009) Melanin-concentrating hormone neurons discharge in a reciprocal manner to orexin neurons across the sleep-wake cycle. Proc Natl Acad Sci USA 106:24182422. CrossRef

Hassani OK, Henny P, Lee MG, Jones BE (2010) GABAergic neurons intermingled with orexin and $\mathrm{MCH}$ neurons in the lateral hypothal- amus discharge maximally during sleep. Eur J Neurosci 32:448457. CrossRef Medline

Hendricks JC, Morrison AR, Mann GL (1982) Different behaviors during paradoxical sleep without atonia depend on pontine lesion site. Brain Res 239:81-105. Medline

Ibata K, Sun Q, Turrigiano GG (2008) Rapid synaptic scaling induced by changes in postsynaptic firing. Neuron 57:819-826. CrossRef Medline

Jego S, Glasgow SD, Herrera CG, Ekstrand M, Reed SJ, Boyce R, Friedman J, Burdakov D, Adamantidis AR (2013) Optogenetic identification of a rapid eye movement sleep modulatory circuit in the hypothalamus. Nat Neurosci 16:1637-1643. CrossRef Medline Jones BE (2017) Principal cell types of sleep-wake regulatory circuits. Curr Opin Neurobiol 44:101-109. CrossRef Medline

Jouvet M (1962) Recherches sur les structures nerveuses et les mécanismes responsables des differentes phases du sommeil physiologique. Arch Ital Biol 100:125-206.

Kaur S, Thankachan S, Begum S, Liu M, Blanco-Centurion C, Shiromani PJ (2009) Hypocretin-2 saporin lesions of the ventrolateral periaquaductal gray (vIPAG) increase REM sleep in hypocretin knockout mice. PLoS One 4:e6346. CrossRef Medline

Kilman V, van Rossum MC, Turrigiano GG (2002) Activity deprivation reduces miniature IPSC amplitude by decreasing the number of postsynaptic $\mathrm{GABA}(\mathrm{A})$ receptors clustered at neocortical synapses. J Neurosci 22:1328-1337. Medline

Konadhode RR, Pelluru D, Blanco-Centurion C, Zayachkivsky A, Liu M, Uhde T, Glen WB Jr, van den Pol AN, Mulholland PJ, Shiromani PJ (2013) Optogenetic stimulation of $\mathrm{MCH}$ neurons increases sleep. J Neurosci 33:10257-10263. CrossRef

Lee MG, Hassani OK, Jones BE (2005) Discharge of identified orexin/ hypocretin neurons across the sleep-waking cycle. J Neurosci 25:6716-6720. CrossRef Medline

Levey Al, Kitt CA, Simonds WF, Price DL, Brann MR (1991) Identification and localization of muscarinic acetylcholine receptor proteins in brain with subtype-specific antibodies. J Neurosci 11: 3218-3226. Medline

Lu J, Sherman D, Devor M, Saper CB (2006) A putative flip-flop switch for control of REM sleep. Nature 441:589-594. CrossRef Medline

Maloney KJ, Mainville L, Jones BE (1999) Differential c-Fos expression in cholinergic, monoaminergic and GABAergic cell groups of the pontomesencephalic tegmentum after paradoxical sleep deprivation and recovery. J Neurosci 19:3057-3072. Medline

Maloney KJ, Mainville L, Jones BE (2000) c-Fos expression in GABAergic, serotonergic and other neurons of the pontomedullary reticular formation and raphe after paradoxical sleep deprivation and recovery. J Neurosci 20:4669-4679. Medline

Marty S, Wehrlé R, Fritschy JM, Sotelo C (2004) Quantitative effects produced by modifications of neuronal activity on the size of $\mathrm{GABA}_{\mathrm{A}}$ receptor clusters in hippocampal slice cultures. Eur $\mathrm{J}$ Neurosci 20:427-440. CrossRef Medline

Modirrousta M, Mainville L, Jones BE (2005) Orexin and MCH neurons express c-Fos differently after sleep deprivation vs. recovery and bear different adrenergic receptors. Eur J Neurosci 21:28072816. CrossRef Medline

Modirrousta M, Mainville L, Jones BE (2007) Dynamic changes in GABAA receptors on basal forebrain cholinergic neurons following sleep deprivation and recovery. BMC Neurosci 8:15. CrossRef Medline

Nishino S, Mignot E (1997) Pharmacological aspects of human and canine narcolepsy. Prog Neurobiol 52:27-78. Medline

Nishino S, Mignot E (2011) Narcolepsy and cataplexy. Handb Clin Neurol 99:783-814. CrossRef Medline

Nusser Z, Hájos N, Somogyi P, Mody I (1998) Increased number of synaptic GABA(A) receptors underlies potentiation at hippocampal inhibitory synapses. Nature 395:172-177. CrossRef Medline

Onoe H, Sakai K (1995) Kainate receptors: a novel mechanism in paradoxical (REM) sleep generation. Neuroreport 6:353-356. Medline 
Paxinos G, Franklin KBJ (2001) The mouse brain in stereotaxic coordinates, Ed 2. San Diego: Academic Press.

Petitjean F, Sakai K, Blondaux C, Jouvet M (1975) [Hypersomnia by isthmic lesion in cat. II. Neurophysiological and pharmacological study]. Brain Res 88:439-453. Medline

Puentes-Mestril C, Aton SJ (2017) Linking network activity to synaptic plasticity during sleep: hypotheses and recent data. Front Neural Circuits 11:61. CrossRef Medline

Sakai K (1980) Some anatomical and physiological properties of pontomesencephalic tegmental neurons with special reference to the PGO waves and postural atonia during paradoxical sleep in the cat. In: The reticular formation revisited (Hobson JA, Brazier MAB, eds), pp 427-447. New York: Raven Press.

Sakai K, Koyama Y (1996) Are there cholinergic and non-cholinergic paradoxical sleep-on neurones in the pons? Neuroreport 7:24492453. Medline

Sanford LD, Tang X, Xiao J, Ross RJ, Morrison AR (2003) GABAergic regulation of REM sleep in reticularis pontis oralis and caudalis in rats. J Neurophysiol 90:938-945. CrossRef Medline

Sapin E, Lapray D, Bérod A, Goutagny R, Léger L, Ravassard P, Clément O, Hanriot L, Fort P, Luppi PH (2009) Localization of the brainstem GABAergic neurons controlling paradoxical (REM) sleep. PLoS One 4:e4272. CrossRef Medline

Sastre JP, Buda C, Kitahama K, Jouvet M (1996) Importance of the ventrolateral region of the periaqueductal gray and adjacent tegmentum in the control of paradoxical sleep as studied by muscimol microinjections in the cat. Neuroscience 74:415-426. Medline

Timofeev I, Chauvette S (2017) Sleep slow oscillation and plasticity. Curr Opin Neurobiol 44:116-126. CrossRef Medline

Tononi G, Cirelli C (2003) Sleep and synaptic homeostasis: a hypothesis. Brain Res Bull 62:143-150. CrossRef

Toossi H, Del Cid-Pellitero E, Jones BE (2016) GABA receptors on orexin and melanin-concentrating hormone neurons are differentially homeostatically regulated following sleep deprivation. eNeuro 3. CrossRef

Toossi H, Del Cid-Pellitero E, Jones BE (2017) Homeostatic regulation through GABA and acetylcholine muscarinic receptors of motor trigeminal neurons following sleep deprivation. Brain Struct Funct 222:3163-3178. CrossRef

Tsunematsu T, Ueno T, Tabuchi S, Inutsuka A, Tanaka KF, Hasuwa H, Kilduff TS, Terao A, Yamanaka A (2014) Optogenetic manipulation of activity and temporally controlled cell-specific ablation reveal a role for $\mathrm{MCH}$ neurons in sleep/wake regulation. J Neurosci 34:6896-6909. CrossRef Medline

Turrigiano GG (1999) Homeostatic plasticity in neuronal networks: the more things change, the more they stay the same. Trends Neurosci 22:221-227. Medline

Turrigiano GG, Leslie KR, Desai NS, Rutherford LC, Nelson SB (1998) Activity-dependent scaling of quantal amplitude in neocortical neurons. Nature 391:892-896. CrossRef Medline

Vanni-Mercier G, Sakai K, Lin JS, Jouvet M (1989) Mapping of cholinoceptive brainstem structures responsible for the generation of paradoxical sleep in the cat. Arch Ital Biol 127:133-164. Medline

Velazquez-Moctezuma J, Gillin JC, Shiromani PJ (1989) Effect of specific M1, M2 muscarinic receptor agonists on REM sleep generation. Brain Res 503:128-131. Medline

Vyazovskiy VV, Cirelli C, Pfister-Genskow M, Faraguna U, Tononi G (2008) Molecular and electrophysiological evidence for net synaptic potentiation in wake and depression in sleep. Nat Neurosci 11:200-208. CrossRef Medline

Wan Q, Xiong ZG, Man HY, Ackerley CA, Braunton J, Lu WY, Becker LE, MacDonald JF, Wang YT (1997) Recruitment of functional GABA(A) receptors to postsynaptic domains by insulin. Nature 388:686-690. CrossRef Medline

Weber F, Chung S, Beier KT, Xu M, Luo L, Dan Y (2015) Control of REM sleep by ventral medulla GABAergic neurons. Nature 526: 435-438. CrossRef Medline

Webster HH, Jones BE (1988) Neurotoxic lesions of the dorsolateral pontomesencephalic tegmentum-cholinergic cell area in the cat. II. Effects upon sleep-waking states. Brain Res 458:285-302. Medline

Xi MC, Morales FR, Chase MH (1999) Evidence that wakefulness and REM sleep are controlled by a GABAergic pontine mechanism. J Neurophysiol 82:2015-2019. CrossRef Medline

Xie M, Li C, He C, Yang L, Tan G, Yan J, Wang J, Hu Z (2016) Short-term sleep deprivation disrupts the molecular composition of ionotropic glutamate receptors in entorhinal cortex and impairs the rat spatial reference memory. Behav Brain Res 300:70-76. CrossRef Medline

Yamanaka T, Him A, Cameron SA, Dutia MB (2000) Rapid compensatory changes in GABA receptor efficacy in rat vestibular neurones after unilateral labyrinthectomy. J Physiol 523:413424. Medline 